\title{
Srijela-an Herbal Preparation with Galactogenic Property
}

\section{Md. Shahidullah', Md. Sallahuddin ${ }^{1}$, Touhida Ahsan', Monira Ahsan ${ }^{3}$, Md. Mustafizur Rahman ${ }^{4}$, Sheikh Nazrul Islam,"}

${ }^{1}$ Ahad Homeo Hall, Jamia Market, Station Road, Kishorgonj, Bangladesh

${ }^{2}$ Department of Gynaecology and Obstetrics, Ibn Sina Medical College, Kalanpur, Dhaka-1212, Bangladesh

${ }^{3}$ Department of Pharmaceutical Chemistry, Faculty of Pharmacy, University of Dhaka, Dhaka-1000, Bangladesh

${ }^{4}$ Department of Pharmacy, Manarat International University, Dhaka-1212, Bangladesh

${ }^{5}$ Institute of Nutrition and Food Science, University of Dhaka, Dhaka-1000, Bangladesh

\section{Email address:}

shahid.ullah294@gmail.com (Md. Shahidullah),drtouhidam15@yahoo.com (T. Ahsan), ahsanm80@yahoo.com (M. Ahsan), md.mustafiz379@gmail.com (Md. Mustafizur Rahman), sheikhnazru109@gmail.com (S. N. Islam), sheikhnazrul@du.ac.bd (S. N. Islam)

\section{To cite this article:}

Md. Shahidullah, Md. Sallahuddin, Touhida Ahsan, Monira Ahsan, Md. Mustafizur Rahman, Sheikh Nazrul Islam. Srijela-an Herbal Preparation with Galactogenic Property. American Journal of Clinical and Experimental Medicine. Vol. 3, No. 2, 2015 , pp. 59-61. doi: 10.11648/j.ajcem.20150302.12

\begin{abstract}
Intake of galactogogues increases breast milk secretion. Aim of this study was to investigate the Srijela (an herbal preparation) for its galactogenic property on postpartum mothers. The study was conducted on 75 postpartum mothers comprising 50 mothers who received Srijela preparation and 25 mothers as control who did not received the Srijela. The Srijela is an herbal preparation of Ashwagandha, Blackseeds, Amlaki, Methi and Hing. Observations were made on lactation factorstime of starting milk secretion after delivery, frequency of breast feeding, quantity of milk secretion, frequency of urination and weight gain of the babies. It was seen that Srijela enhanced the breast milk secretion in terms of onset, frequency, and quantity of milk secretion in the postpartum mothers as compared to those who did not received Srijela. Frequency of urination (an indicator for increase milk intake) was also remarkably higher for the babies of Srijela mothers than that for the babies of nonSrijela mothers. Weight gain of babies was also higher for Srijela mothers. This study indicated that Srijela promoted breast milk secretion in postpartum mothers.
\end{abstract}

Keywords: Srijela, Herbal Preparation, Galactogogues, Postpartum Mothers

\section{Introduction}

Breast-feeding should start from the very first day of birth. It facilitates healthy galactogenesis in the mother. Breast milk is the best source of nutrition for newborns. Sustained breast feeding has endless benefits for the baby but insufficient milk supply discontinues breastfeeding [1-3]. Poor lactation affects neonatal growth and development. Galactagogues enhance breast milk secretion. It assists initiation, maintenance, or augmentation of the rate of maternal milk production and secretion. Human milk production is a complex physiologic process involving physical and emotional factors, and interaction of multiple hormones, the most important one is prolactin. During pregnancy and lactation, hormonal changes trigger lactogenesis of the mammary lactocytes [4]. Prolactin synthesizes breast milk and oxytocin ejects the milk from the breast.

Many plant galactogogues are in use to promote lactation
[5-13]. Srijela is an herbal galactogogue. It is a preparation of Ashwagandha, Blackseeds, Amlaki, Methi and Hing. The constituents of Srijela have been reported to possess galactogogue principles and enhance breast milk secretion [5, $8,12,14]$. Reports on the trdaditional or ayurvedic use of herbal products as galactogogue are many, but scientific or clinical research is rare. This study has investigated galactogogue action of Srijela on postpartum mothers.

\section{Materials and Methods}

\subsection{Study Population}

This is a case control cross sectional study conducted on seventy five postpartum mothers of 18 to 35 years age with primi to multi gravida living in semi urban location. Fifty postpartum mothers were taken as case subject who received Srijela preparation and twenty five mothers were used as control who did not take Srijela. The case subjects afforded 
exclusive breastfeeding to their newborns while the control mothers gave complement feeding along with breastfeeding to the newborns. Ethical approval was obtained from Ethical Committee comprising 15 members of the Biological Faculty of the University of Dhaka. Consent was taken from the postpartum mothers receiving the Srijela preparation.

Srijela is a preparation of commonly used spices, minor fruit and medicinal plant. The composition of Srijela preparation included- aqueous extracts of 9\% Ashwagandha (Withania somnifera), 2\% Blackseed (Nigella sativa), 4\% Amlaki (Emblica Officinalis), 1\% Methi (Trigonella foenumgraecum) and 1\% Hing (Ferula assa-foetida).

\subsection{Observation}

Srijela preparation was given as: one teaspoonful $(5 \mathrm{ml})$ thrice daily for 30 days to the 50 postpartum mothers. The control postpartum mother did not receive Srijela. Observations were made on the time of onset of milk secretion after delivery, frequency of breast feeding, quantity of milk secretion, frequency of urination of babies and weight gain of the babies.

\subsection{Statistically Analysis}

SPSS software package (version 20.0, IBM SPSS Inc.
Chicago, USA) was used to analyze the data. Descriptive statistics were calculated for all variables. Independent sample test was performed to find the statistical variation in the glactogenic parameters between the Srijela and non Srijela mothers.

\section{Results \& Discussion}

It was resulted in that Srijela supplementation to postpartum mothers enhanced breast milk secretion. It eloquently increased the onset, frequency and quantity of breast milk secretion. Observation showed that there have significantly $(\mathrm{p}<0.05)$ faster onset of milk secretion (13.90 \pm 1.75 hour) after delivery, higher frequency of milk secretion ( $8.32 \pm 0.89$ times/day) and increased quantity of milk output $(416.70 \pm 44.52 \mathrm{ml} /$ day approximately) in the Srijela mothers, while start of milk secretion, rate of milk outcome and volume of milk production were $27.76 \pm 7.16$ hour, $5.12 \pm 1.64$ times/day and $256 \pm 82.06 \mathrm{ml} /$ day approximately respectively in the control postpartum mothers. Frequency of urination $(8.32 \pm 1.28$ versus $4.96 \pm 0.93$ times/day) and weight gain $(8.38 \pm 0.75 \mathrm{Ib}$ versus $7.06 \pm 0.22$ Ib after 30 days were also found significantly $(\mathrm{p}<0.05)$ higher in Srijela babies than those in the non-Srijela babies.

Table 1. Lactation outcomes between Srijela receiving and Control postpartum mothers

\begin{tabular}{llll}
\hline Lactation factor & Srijela group & Control group & p value \\
\hline Time of starting milk secretion after delivery (hour) & $13.90 \pm 1.75$ & $27.76 \pm 7.16$ \\
Frequency of breast feeding/ day & $8.32 \pm 0.89$ & $5.12 \pm 1.64$ \\
Quantity of milk secretion (ml)/day & $416.70 \pm 44.52$ & $256 \pm 82.06$ & $4.96 \pm 0.93$ \\
Frequency of urination/ day & $8.32 \pm 1.28$ & $7.06 \pm 0.22$ \\
Weight of baby after 30 days & $8.38 \pm 0.75$ & & P \\
Weight increment after 30 days & & \\
\hline
\end{tabular}

$\mathrm{p}=$ level of significance at $95 \%$ confidence $(\mathrm{p}<0.05)$

Breast milk is the best food for the babies. The very first breast milk- the colostrum, is the gold nutrition for the neonate. It helps healthy growth and development of cognitive function and immunity. Colostrum is the first defense for neonates. It stimulates the newborn's immune system [15]. The human colostrum and early milk have unique anti-infective properties against bacterial and viral pathogens. These are rich in immune components including immunoglobulins, predominantly $\operatorname{IgA}$, and viable immune cells such as lymphocytes, macrophages, neutrophils, antioxidant micronutrients, and non-immune bioactive compounds such as lactoferrin, lysozyme, and transferrin. Even more, it contains certain antigen-specific antibodies against both bacteria and viruses $[15,16]$.

Adequate breast milk supply is the utmost need for normal

functioning of the newborns. Lack of knowledge on breastfeeding benefit, illness, use of contraceptive, malnutrition, poverty and even genetic factor can result in poor lactation. Any delay on the onset of milk production and ejection in the first days after delivery can cause breast- feeding failure [17]. Some lactating mothers use dopamine antagonist such as domperidone as galactagogues to increase prolactin level [18], but it has frequent side effect that makes it unacceptable for the lactating mothers. Natural herbal galactagogues do not have side effects. Srijela is a natural product prepared from the certain herbs, which are frequently consumed by the postpartum mothers.

As a galactogogue, Srijela preparation showed significant increase of milk secretion to the postpartum mothers. It induced onset of milk secretion at $13.90 \pm 1.75$ hour postpartum, which was much faster as compared to the control and as reported by others [17,19]. Frequency of breast milk feed was recorded more than 8 times per day and volume of milk secretion was about $416.70 \pm 44.52$ $\mathrm{ml} /$ day with Srijela, which were equivalent to reported data [20,21], but much higher than the control postpartum mothers. Frequency of urination and weight gain of the neonates of Srijela mothers were also found high as compared to those of non-Srijela mothers and corresponded to exclusive breastfeeding neonates $[20,21]$. 


\section{Conclusion}

Srijela - an herbal preparation is indicated as an effective galatagogue. It actively enhances human breast milk secretion. It speeds up onset of breast milk ejection along with increasing its production and secretion. Routine use of Srijela by postpartum mother would help management of poor lactation and thus, contribute to healthy growth and development of the neonates.

\section{Acknowledgement}

Authors thank the postpartum mothers for voluntary participation in carrying out this research. Also thank is given to the Ahad Homeo Hall for supplying the Srijela preparation.

\section{References}

[1] Hauck Y.L., Fenwick J., Dhaliwal S.S., Butt J. A Western Australian survey of breastfeeding initiation, prevalence and early cessation patterns. Matern Child Health J, 2011. Vol2. Pp.260-268.

[2] Lewis J.A. Maternal perceptions of insufficient milk supply in breastfeeding. Am J Matern Child Nurs, 2009. Vol.34. p.264.

[3] Li R., Fein S.B., Chen J., et al. Why mothers stop breastfeeding: Mothers' self-reported reasons for stopping during the first year. Pediatrics, 2008. Vol.122(Suppl 2). Pp.S69-S76.

[4] Linzell J.1., Peaker M. Mechanism of milk secretion. Physiological Reviews, 1971. Vol.51(3). Pp.564-597.

[5] Reeder C., Legrand A., O'Conner-Von S. The Effect of Fenugreek on Milk Production and Prolactin Levels in Mothers of Premature Infants. J Human Lactation, 2011. Vol.27(1). p.74.

[6] Nice, F.J. Common Herbs and Foods Used as Galactogogues, ICAN: Infant, Child \& Adolescent Nutrition, 2011. Vol.3(3). pp.129-132.

[7] Sayed N.Z., Deo R., Mukundan U. Herbal remedies used by Warlis of Dahanu to induce lactation in nursing mothers. Indian J Tradit Knowl., 2007. Vol.6. Pp.602-605.

[8] Swafford S., Berens B. Effect of fenugreek on breast milk production. Annual meeting abstracts. ABM News and Views, 2000. Vol.6(3). Pp10-12.
[9] Pattnaik A.K. Effect of herbal additives on lactating ruminants with or without subclinical mastitis, Thesis submitted to Orissa University of Agriculture and Technology, Bhubaneswar (Orissa) India, 2003.

[10] Bingel A.S., Farmsworth M.R. Higher plants as potential sources of galactagogues. Econ. Med. Plant Res. 1991. Vol.6. Pp.1-54.

[11] Kirtikar K.P., Basu .BD. Effect of herbal preparation on hypogalactic cross breed cows. In: Indian Medicinal Plants, 2nd Edn, Periodical Express, New Delhi, 1975.

[12] Agrawala I.P., Achar M.V., Boradkar R.V., Roy N. Galactagogue action of Cuminum cyminum and Nigella sativa. Indian J Med Res., 1968. Vol.56 pp.841-844.

[13] Chopra R.N., Nayar S.L., Chopra I.C. Effect of some herbal preparations as galactogogue in milch animals. Glossary of Indian Medicinal Plants, CSIR Publication, New Delhi, 1956.

[14] Acharya D. Herbs from India's Tribal Pouch: Ashwagandha. 2012:http://www.greenmedinfo.com/blog/ herbs-indias-tribalpouch-ashwagandha.

[15] Islam S.N., Ahmed L., Khan M.N.I., Huque S., Begum A., Yunus A.B.M. Immune components (IgA, IgM, IgG, immune cells) of colostrums of Bangladeshi mothers. Pediartric Int. 2006. Vol.48(6). Pp.549-554.

[16] Ahmed L., Khan M.N.I., Huque S., Ahsan M. Antioxidant micronutrient profile (vitamin E, C, A, copper, zinc, iron) of colostrum: association with maternal characteristics. J Trop Pediatr, 2004. Vol.50(6). Pp.357-358.

[17] Sakha K., Behbahan A.G.G. The onset time of lactation after delivery. Medical journal of the Islamic Republic of Iran, 2005. Vol. 19 (2). Pp.135-139.

[18] Da Silva O.P., Knoppert D.C., Angelini M.M., et al. Effect of domperidone on milk production in mothers of premature newborns: A randomized, double-blind, placebo-controlled trial. Can Med Assoc J, 2001. Pp.164:17-21.

[19] Neville M.C.. Milk secretion: an overview. http://mammary.nih.gov/reviews/lactation/Neville001/ index.html\#biblio.

[20] Low milk supply/BabyCenter. http://www.babycenter.com/0_low-milk-supply_8487.bc

[21] Low milk supply. http://www.thewomens.org.au/ Lowmilksupply. 